\title{
The Femoral and Lateral Femoral Cutaneous Nerve Palsy After Periacetabular Osteotomy Operation
}

\author{
Ismail Boyraz ${ }^{\mathrm{a}, \mathrm{c}}$, Hakan Sarman ${ }^{\mathrm{b}}$, Bunyamin Koc ${ }^{\mathrm{a}}$, Cengiz Isik ${ }^{\mathrm{b}}$, Hilal Caglar ${ }^{\mathrm{a}}$
}

\begin{abstract}
Acetabular dysplasia is a complex multi-plane deformity of the acetabulum, which results in the development of osteoarthritis at an early age in young adults. A female patient at age of 24 was admitted to our rehabilitation clinic because of hip pain and weakness. The patient had had bilateral hip joint dysplasia and she had been operated from right side on December 25, 2013 by Ganz tecnique which is called periacetabular osteotomy. After the surgery, the patient had limitation of hip joint and limping. When the patient was admitted to our clinic, the patient's hip muscles force was $2 / 5$, knee muscles force was 5/5 and she has hypoesthesia at L2-3 dermatomes. Her EMG result exposed femoral and lateral femoral cutaneous nerve injury. The patient could walk with a canedien. We took the patient for rehabilitation treatment to strengthen her muscles and to decrease hip pain and hip limitation. The aims of treatment for acetabular dysplasia are the normalization of the relationship between the femoral head and acetabulum on weight bearing position, and to provide a painless, stable, and functional hip in the long term. There may be several complications after hip surgery such as nerve or vessel injury, acetabular osteonecrosis, arthrosis, intraarticular osteotomy, heterotopic ossification, pulmonary emboli, deep vein thrombosis (DVT), and RSDS (algoneurodistrofia).
\end{abstract}

Keywords: Acetabuluar dysplasia; Nerve palsy; Osteotomy

\section{Introduction}

Acetabular dysplasia is a complex multi-plane deformity of the

Manuscript accepted for publication January 05, 2015

${ }^{a}$ Bolu Abant Izzet Baysal University Medical School, Department of Physical Medicine and Rehabilitation Training and Research Hospital Bolu, Turkey bBolu Abant Izzet Baysal University Training and Research Medical School, Department of Orthopedics, Bolu, Turkey

${ }^{\mathrm{c} C o r r e s p o n d i n g ~ A u t h o r: ~ I s m a i l ~ B o y r a z, ~ B o l u ~ A b a n t ~ I z z e t ~ B a y s a l ~ U n i v e r s i t y ~}$ Medical School, Department of Physical Medicine and Rehabilitation, AIBU Physical Therapy and Rehabilitation Training and Research Hospital, Karacasu, Bolu, Turkey. Email: boyraz@yahoo.com

doi: http://dx.doi.org/10.14740/jmc2033w acetabulum, which results in the development of osteoarthritis at an early age in young adults. Developmental dysplasia of the hip (DDH), formerly known as congenital dislocation of the hip (CDH) comprises a spectrum of abnormalities of the hip in infants and children that includes an immature hip, acetabular dysplasia with or without dislocation, subluxation of the femoral head as well as being dislocatable and subluxable. Although some of these changes may resolve spontaneously, if not diagnosed or monitored during infancy, the hip will worsen with time. As some reports have documented, dislocation or dysplasia of the hip can occur after a normal neonatal screening examination. Presently, main methods for screening include clinical examination and ultrasound. Pelvic radiograph has been used after 4 months of age to assure bone congruency. Among the risk factors investigated, the present findings suggest that the most significant risk factors associated with DDH include those presenting in the breech position during delivery, being female, having an affected left hip, or having a family history of DDH [1]. A significant association between primiparity and DDH was found in the case-control studies [2]. The diagnosis can be difficult, even in experienced hands, and a late diagnosis does not necessarily imply a missed diagnosis. Treatment depends on the age of initial diagnosis and success of previous treatment. Earlier treatment usually requires less aggressive methods. Follow-up should be continued to skeletal maturity, and both hips should be carefully evaluated [3]. As hip arthroscopy continues to be performed more commonly, it is very important to accurately diagnose the pathomorphology preoperatively to minimize the failure rates after joint preservation surgery [4].

We diagnosed nerve injuries at the young patient after the periacetabular osteotomy surgery and she was admitted to our clinic for rehabilitation treatment. Our patient's EMG result was at odds with her clinic examination. We must be carefully examine the patients after the periacetabular osteotomy surgery as there may be multiple nerve damage.

\section{Case Report}

A female patient at age of 24 was admitted to our rehabilitation clinic because of hip pain and weakness. The patient had had bilateral hip joint dysplasia and she had been operated from right side on December 25, 2013 by Ganz tecnique which is 
called periacetabular osteotomy. After the surgery, the patient had limitation of hip joint and limping. She could start to walk at age 1 and had imbalance during walking. Bilatheral DKC was diagnosed by the doctor and spica cast was applied for 4 months. She was placed into a hip abduction brace for 1 year. She never had any hip pain until 22 years old. The patient fell down stairs on the hip and then she had coxigeal pain. She suffered bilatheral hip pain especially much more on the right side increasing by moving since 2013 . The patient was operated on December 25, 2013 by orthopeadic surgeon beause of hip joint tear and bone crack.

The patient has hip pain and weakness when she turned to our hospital. When the patient was examined at the clinic, the patient's hip internal and external rotation was limited at $30^{\circ}$ and hip muscles force was $2 / 5$. ROM of knee was full and knee muscles force was $5 / 5$ and she had hypoesthesia at L2-3 dermatomes. Patella reflex diminished; Trendelenburg sign was positive at the right side. MRI scan had been obtained before surgery and the result was bilatheral hip displasia much marked at right hip, thick and degenerated labrum at the right side, derangement due to degeneration at the left hip labrum. Her EMG result exposed femoral nerve which had partial axonal injury and severe lateral femoral cutaneous nevre injury. Femoral nerve had low motor amplitude, the femoral lateral cutaneous nerve could not obtain from right side. There were severe partial denervation along with severe neurogenic involvement in needle EMG measurement of the rectus femoris muscle and light neurogenic changes in the other muscles innervated by femoral nerve. The patient could walk with a canedien. We took the patient for rehabilitation treatment to strengthen her muscles and to decrease hip pain and hip limitation.

\section{Discussion}

Our patient has femoral and the lateral cutaneous nerve injury after surgery. EMG revealed and corrected these findings but the patient has weakness at the gluteal muscles. She may have several nerve lesions innervated gluteal muscles. It may be that EMG cannot reveal this injury due to examination failure. The aims of treatment for acetabular dysplasia are the normalization of the relationship between the femoral head and acetabulum on weight bearing position, and to provide a painless, stable, and functional hip in the long term. There may be several complications after hip surgery such as nevre or vessel injury, acetabular osteonecrosis, arthrosis, intraarticular osteotomy, heterotopic ossification, pulmonary emboli, deep vein thrombosis (DVT), and RSDS (algoneurodistrofia).

The Bernese periacetabular osteotomy (PAO) as described by Ganz et al has become the preferred pelvic osteotomy in many centers for the treatment of symptomatic acetabular dysplasia in the young adult. One of the major potential complications associated with the procedure is nerve injury. In the first report on the PAO, Ganz et al reviewed 75 cases and reported one femoral neurapraxia with no injuries to the sciatic nerve [5]. Davey and Santore noted four sciatic or peroneal nevre palsies in their first 70 patients and discussed the lengthy learning curve associated with the procedure [6]. Subsequent studies of the osteotomy using a modern abductor-sparing approach also had femoral and sciatic nerve palsies at rates ranging from $0 \%$ to $15 \%$ [5]. Sierra et al found an incidence of major nerve injury after PAO of $2.1 \%$ in more than 1,700 PAOs performed in five centers. They were unable to determine which patients are at increased risk of nerve injury at the time of PAO. Femoral nevre injuries have a good prognosis whereas the degree of neurologic recovery of sciatic nerve injuries depends on the cause and severity of the damage [7]. In particular the lateral cutaneous nerve is prone to injury reportedly in up to one-third of patients but regarded as a minor problem and given only little attention by the surgeon. The modified Smith-Petersen approach is likely to lead to division, especially of the first branch of the lateral cutaneous nerve but dysaesthesias of the lateral femoral cutaneous nerve were also observed frequently for the classic Smith-Petersen approach [8].

Current efforts for prevention of hip dysplasia are primarily focused on early detection and early intervention to avoid long-term consequences of neglected hip dysplasia. Better prevention may be possible by decreasing postnatal environmental factors that influence the development of hip dysplasia. Nerve injuries after surgey must be diagnosed as early as possible and the patient must be evaluated for rehabilitation treatment.

\section{Conflict of Interest}

All authors have no conflict of interest.

\section{Disclosure}

All authors have no financial disclosures. We do not have any grant.

\section{References}

1. Ortiz-Neira CL, Paolucci EO, Donnon T. A meta-analysis of common risk factors associated with the diagnosis of developmental dysplasia of the hip in newborns. Eur J Radiol. 2012;81(3):e344-351.

2. de Hundt M, Vlemmix F, Bais JM, Hutton EK, de Groot CJ, Mol BW, Kok M. Risk factors for developmental dysplasia of the hip: a meta-analysis. Eur J Obstet Gynecol Reprod Biol. 2012;165(1):8-17.

3. McCarthy J, Scoles PV, MacEwen GD. Developmental dysplasia of the hip (DDH). Current Orthopaedics. 2005;19:223-230.

4. Ross JR, Clohisy JC, Baca G, Sink E. Patient and disease characteristics associated with hip arthroscopy failure in acetabular dysplasia. J Arthroplasty. 2014;29(9 Suppl):160-163.

5. Ganz R, Klaue K, Vinh TS, Mast JW. A new periacetabular osteotomy for the treatment of hip dysplasias. Technique and preliminary results. Clin Orthop Relat Res. 1988;(232):26-36. 
6. Davey JP, Santore RF. Complications of periacetabular osteotomy. Clin Orthop Relat Res. 1999;(363):33-37.

7. Sierra RJ, Beaule P, Zaltz I, Millis MB, Clohisy JC, Trousdale RT. Prevention of nerve injury after periacetabular osteotomy. Clin Orthop Relat Res. 2012;470(8):2209-
2219.

8. Biedermann R, Donnan L, Gabriel A, Wachter R, Krismer M, Behensky H. Complications and patient satisfaction after periacetabular pelvic osteotomy. Int Orthop. 2008;32(5):611-617. 